\title{
A collaborative software inspection model for a small scale software house in a developing economy
}

\author{
Olalekan S. Akinola \\ Department of Computer Science, University of Ibadan, Nigeria
}

\begin{abstract}
Quality has been a major driving factor for software consumption. This has been seriously compromised among software developers especially in small scale software outfits in developing countries such as Nigeria. The Software development process in these software houses needs to be re-engineered in order to improve the quality of software being produced and to meet the global demand on quality software. One of the ways of improving the quality of software is the incorporation of formal inspection into the software development process. A collaborative software inspection model is proposed in this article. The advantages of the model as well as enabling factors for the model to function properly are also presented.
\end{abstract}

Keywords: Software development, Collaborative software Inspection, Software quality

\section{INTRODUCTION}

Every manufacturer of a product is expected to institute strategies that will assure that his product is of utmost quality. This quality holds for the software manufacturers too, whose product in this context is software. Quality software can be said to be a "software that satisfies the needs of the users and the programmers involved in it", (Pfleeger, 1991).

The primary purpose of a software inspection is to identify defects existing within software work products developed throughout the development process (e.g., user requirements specifications, design documents, code). It is not only used to correct the defect, but also to evaluate and improve the development process itself. Software inspection is a peer review in which a small group of software developers (usually between 3 to 8) examine another software developer's work for bugs (Porter, et al., 1997; Tyran, 2006). By detecting defects early and preventing their leakage into subsequent activities, the need for later detection and rework (which is essential for reduced cycle time and lower cost) is eliminated. Ultimately, software inspection offers value in improving software quality, reliability, availability and maintainability.

Software inspection is widely accepted as a step in the software development process among software developers in developed nations of the world. Chatzigeorgiou and Antoniadis, (2003) report that the number of software organizations that have incorporated formal reviews (inspections) in their development process is constantly increasing and the belief that efficient inspections cannot only detect defects but also reduce cycle time, increases process visibility, improves programmers' capability and lower costs is spreading. Filippo, et al., (2003) also report that software inspections have been adopted for many years by industrial organizations because of their impact on product quality and cost of nonquality. Compared to these reports, the adoption of inspection by the Nigerian software practitioners in their development of software is very low compared to what is obtained in developed nations (Olalekan, et al., 2009).

Inspections are used to determine the quality of the software work product, not the quality of the people who create the product. For this reason, managers are not involved in inspection meetings (NASA, 1993). In addition, the results of inspections are handed over to management either statistically or as outcome of inspections for groups of products. By grouping results this way, managers know the value of inspections process without singling out an author. Using the inspection process to judge the competency of authors bring about less than honest and thorough results, that is, co-workers may be reluctant to point out defects if finding them will result in a poor performance appraisal of a colleague.

Software inspection is usually done by a team of experts (analysts, designers, coders, and testers). As proposed by the originator of the exercise, an inspection team size should not be more than four (Fagan, 1976). Research has shown that the larger the inspection team size, the more costly an inspection will be (Porter, et al., 1997; Votta, 1993). Votta (1993) notes that determining the optimal team 
size for inspections is an important contemporary research issue. Shaheen, et al., (2000) work show that there is no optimum size for software inspection. It is clear that the larger the inspection team size, the more costly an inspection will be. However, it is also the case that the more inspectors who read a given artifact, the more likely it is that more defects will be detected. Therefore, there is a trade-off between cost and effectiveness (Shaheen, et al., 2000).

Previous results obtained from the survey of some Nigerian software practitioners on their perception of software inspection as a means of enhancing software quality assurance showed that personnel overhead cost is a major factor affecting their adoption of inspection in their software development processes (Olalekan, et al., 2009). This also adds to the fact that few software professionals were employed by most of the organizations and there was no clear delineation of roles among the few employed. The survey reveals that with many of the software practitioners covered, the same set of personnel is involved in systems analysis, design, coding and testing. Also results from experimental studies had shown that there is no significant difference in using small number of reviewers/inspectors or large number when carrying out software inspection process (Porter, et al., 1997, Shaheen, et al., 2000, Akinola and Osofisan., 2008). This background informs the model here-under proposed.

The Software Inspection Model: Most software companies in developing countries are small and medium sized enterprises, so called SMEs. In a bid to minimize overhead cost, a one-person distributed, collaborative software inspection tagged Joint Application Design Inspection Model (JADInspection), depicted below in Fig. 1 is proposed for the Nigeria software development environment.

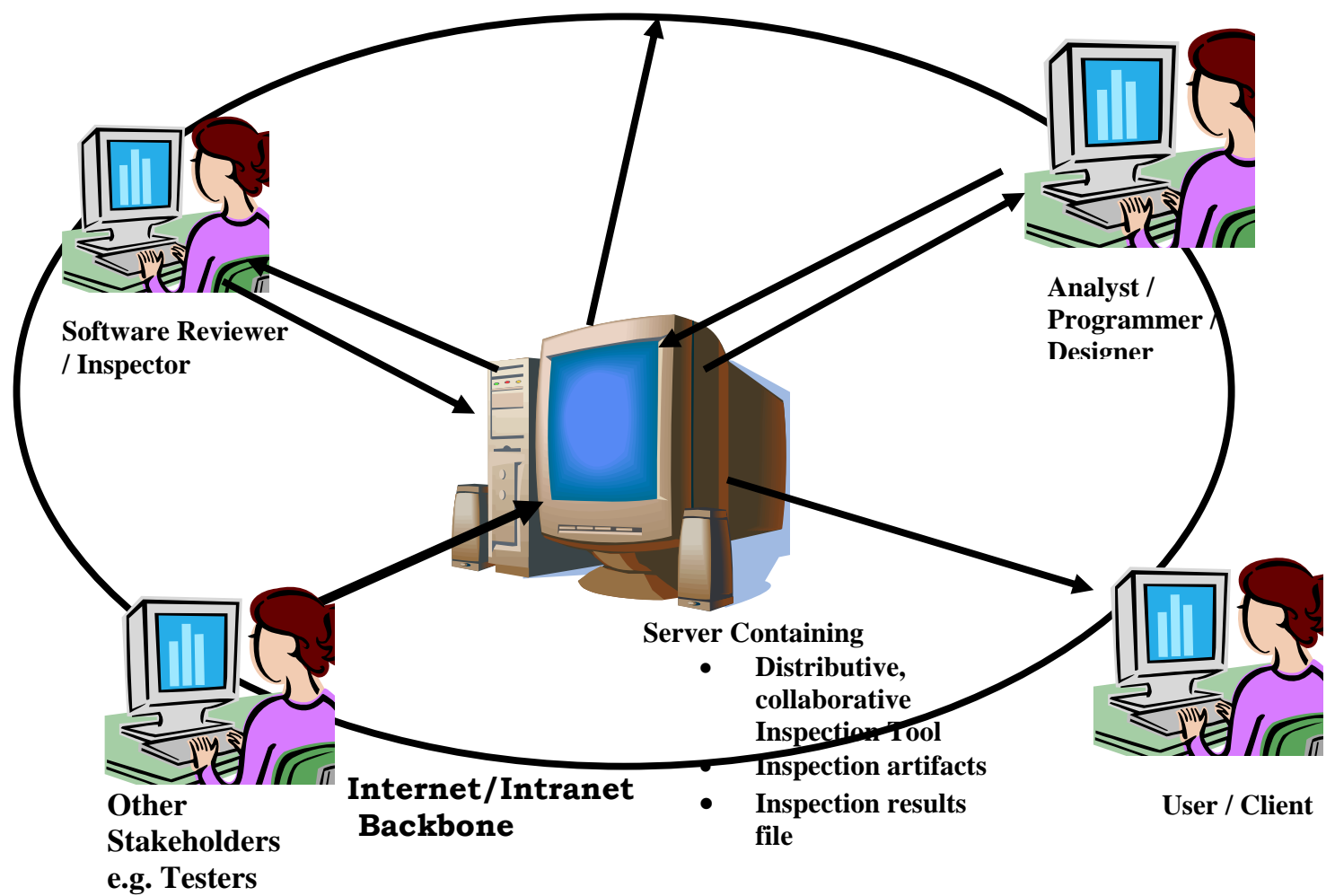

Fig. 1: Joint Application Development- Inspection (JAD-Inspection) Model 
The model depicts a situation where only one experienced software practitioner is employed as software reviewer and / or inspector by a software development house. The reviewer is assumed to have gained a long-term experience in software development processes such as analysis, design and coding for some number of years, at least ten years.

The model assumes that there is an intranet/internet backbone available for the software development house. Possibly, the organization has a recognized web portal on the net. At the central is a web server on which the automated distributed, collaborative inspection tool, the software artifact and inspection results file reside.

The inspector could perform his task real time; as the software artifact is being worked upon by the analyst, designer or programmer depending on the artifact being working on at that time. He could also perform his task at a remote location (possibly at home or inside his car using the modern mobile IT devices). The result of the inspection is sent to a special file on the server or via an email to the software practitioner concerned with the artifact. The designer or programmer can then access the file and use the issues/suggestions raised by the inspector to make corrections to the artifact being developed.

The user /client represented in the model shows that the customer of the artifact can also log on to the server remotely and see the stages of development on the contracted software; so that suggestions on deviation from agreed requirements could be pointed out and corrected at once. In this way, user can also participate in the inspection before final delivery.

Alternatively, a joint meeting of the inspector and other stakeholders including the user/client in form of Joint Application Design (JAD) session as proposed by is summoned to discuss some important fault issues on the software artifact.

The entire model proposed is a kind of joint collaborative, distributed software inspection environment. All the software development stakeholders - Analysts, designers, programmers, software inspectors, testers and the users are represented in the model. The model is distributed in the sense that it is assumed to be deployed on a network using the state-of-the-art technologies intranet/internet and mobile IT devices.

Benefits of this Model: Since project cost is related to the number of inspectors, we have to minimize the number involved in any one. In the original proposed software inspection method, 4 inspectors were the norm and the IEEE standard suggests 3-6 (Porter et al., 1997). If we can reduce to 3 from 4 we save $25 \%$ on traditional Inspections; reducing to 2 we save $50 \%$. If it is possible to perform an Inspection with only one inspector, we could save $75 \%$ of costs of Inspections.

This model is advantageous for the following reasons:

- Personnel overhead cost is highly reduced as only one person is employed as software inspector, and most likely, he is the most senior member of staff in the organization.

- The inspection process will thus be included in the software process of organizations since this aspect of the process is seen to be highly compromized in most of the software houses in Nigeria.

- The quality of the software products being produced by the organization will thus be improved.

- $\quad$ Time and place factor in terms of scheduling an inspection meeting will be eliminated. The inspector logs on to his workstation and brings up the work product to be inspected. $\mathrm{He}$ has all required documents available through the organization's intranet for ancillary and reference documents he may need. He has access to the appropriate checklist, standards, and forms to record any defects found. He can open as many concurrent windows or views with required documents as he needs to perform the Inspection. His time is recorded based on log-on time. When he is through, he submits his Inspection documents to the Producer (designers or coders). He could then make himself available to the Producer if there should be any questions to be trashed as regards the inspection artifact.

- Individual stakeholders could work off-site at his/her own convenience from any location, even at home, especially if the model is implemented on a web portal.

Enabling Factors: The following factors, if provided will enhance the functionality of this proposed model.

(i) Existence of a computer network in the software organization. There must be at least an intranet or a Local Area Network 
(LAN) within the organization. This will foster communication and sharing of software artifact resources among the software development stakeholders in the organization. The organization should endeavour to link the intranet/LAN to the global Internet. The use of Internetenabled mobile telecommunication devices would be made easier in the software development environment.

(ii)

The only software inspector/reviewer must be well-experienced in the process of developing software - analysis, designing and implementation.

Conclusion: A collaborative, internet-enabled software inspection model is proposed in this work. The model is proposed based on minimizing overhead cost of developing software in a small scale software environment. The benefits of the model as well as its enabling factors are presented.

We intend to practically implement and thoroughly test the model for it to be universally accepted as an economic model for software inspection in a small scale software development house situated in a developing economic environment..

\section{REFERENCES}

Akinola, S. Olalekan and Osofisan, O. Adenike (2008). Empirical Study of Factors Affecting the Effectiveness of Software Inspection: A Preliminary Report: European Journal of Scientific Research, vol.19, No. 4, pp. $614-627,2008$.

Chatzigeorgiou A. and Antoniadis, G (2003), "Efficient Management of Inspections In Software Development Projects". Information and Software Technology 45(2003), $671-680$.
Filippo Lanubile, Teresa Mallardo and Fabio Calefato (2003). Tool Support for Geographically Dispersed Inspection Teams, Software Process Improvement And Practice, 8: 217-231 (DOI: 10.1002/spip.184)

Lawrence G. Votta (1993). Does every inspection need a meeting? ACM SIGSoft Software Engineering Notes, 18(5):107-114.

Michael E. Fagan (1976). Design and code inspections to reduce errors in program development. IBM Systems Journal, 15(3):182-211.

National Aeronautics and Space Administration (NASA, 1993). Software Inspection Guidebook, Washington, USA.

Olalekan S. Akinola, Adenike O. Osofisan, Opeoluwa B. Akinkunmi (2009). Industry Perception of the Software Inspection Process: Nigeria Software Industry as a Case Study, African Journal of Computing \& ICT (Journal of the IEEE Nigeria Computer Chapter) Vol. 2, No. 2, Dec. 2009.

Pfleeger S. L. (1991). Software Engineering: The Production of Quality Software, Macmillan Publishing Company, NY, USA.

Porter A. Adam, Harvey, P. Siy, Carol A. Toman and Lawrence G. Votta (1997). An Experiment to Assess the Cost-Benefits of Code Inspections in Large Scale Software development, IEEE Transactions on Software Engineering, vol. 23, No. 6, pp. $329-346$, June 1997.

Shaheen Boodoo, Khaled El Emam, Oliver Laintenberger, and Nazim Madhavji (2000) The Optimal Team Size for UML Design Inspections, National Research Council of Canada, September 2000.

Tyran, Craig K (2006), "A Software Inspection Exercise for the Systems Analysis and Design Course". Journal of Information Systems Education, vol 17(3). 